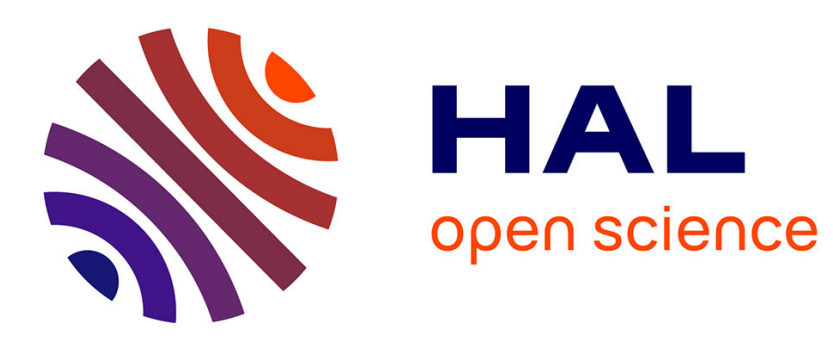

\title{
Lama Bön et maître de maison dans les marches tibétaines de l'Himalaya.
}

\author{
Charles Ramble
}

\section{To cite this version:}

Charles Ramble. Lama Bön et maître de maison dans les marches tibétaines de l'Himalaya.. Une journée dans une vie. Une vie dans une journée. Ascètes et moines aujourd'hui, 2018, pp.59-81. hal-03112114

\section{HAL Id: hal-03112114 \\ https://hal.science/hal-03112114}

Submitted on 2 Feb 2021

HAL is a multi-disciplinary open access archive for the deposit and dissemination of scientific research documents, whether they are published or not. The documents may come from teaching and research institutions in France or abroad, or from public or private research centers.
L'archive ouverte pluridisciplinaire HAL, est destinée au dépôt et à la diffusion de documents scientifiques de niveau recherche, publiés ou non, émanant des établissements d'enseignement et de recherche français ou étrangers, des laboratoires publics ou privés. 


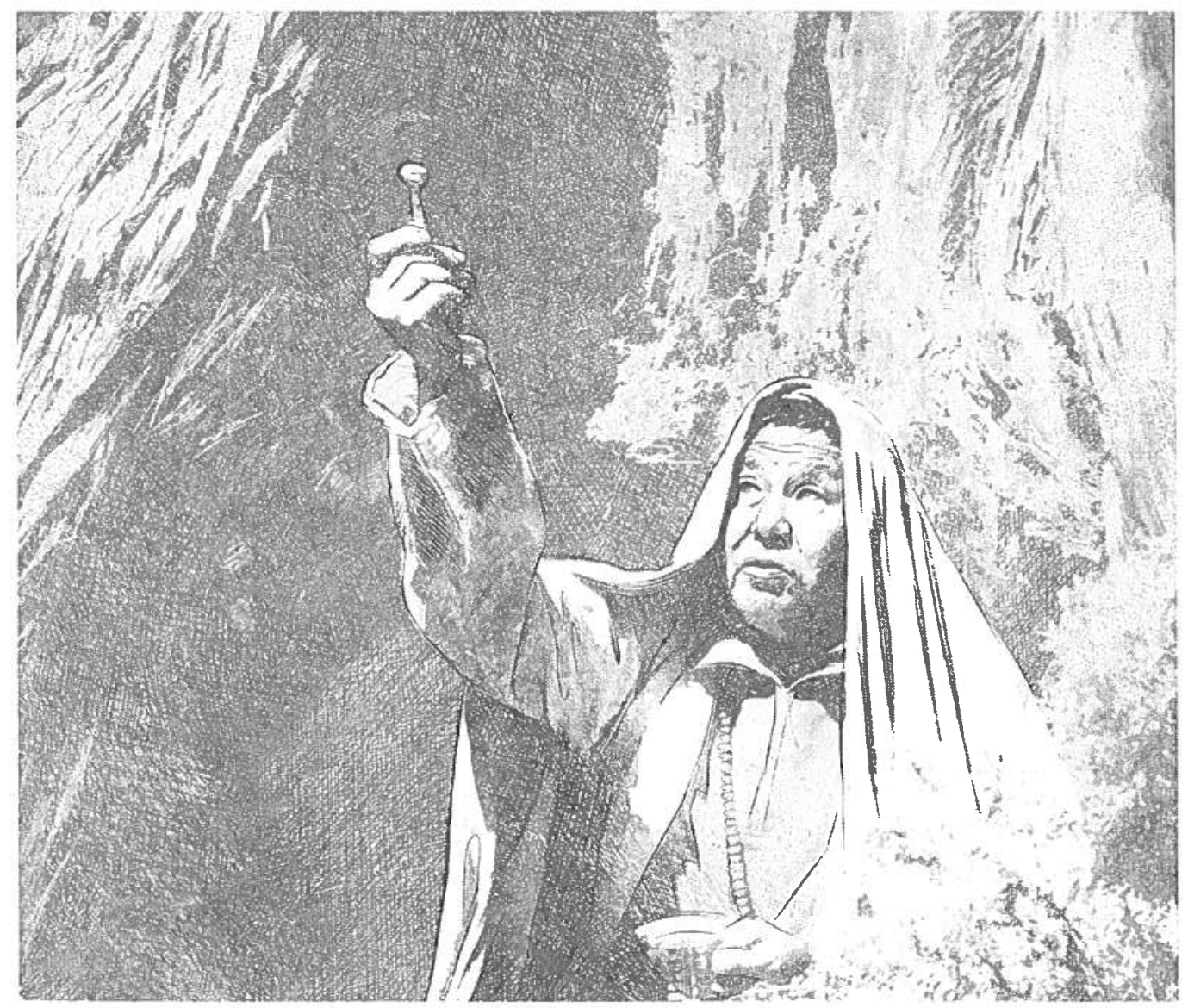




\title{
KHAPTRE \\ Lama Bön et maître de maison dans les marches tibétaines de l'Himalaya
}

$4\}$

\author{
Charles Ramble \\ $\checkmark$ thit siècles de lawar

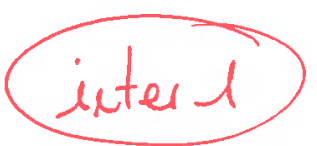 \\ I \\ tous les matins, est de changer l'eau des bols culi se trouvent sur \\ l'autel de son temple domestique. Comme tous les villageois tibé- \\ tains dévots, il sait qu'il faut prier régulièrement les dieux du Mus- \\ tang, un district reculé des montagnes népalaises. C'est le minimum \\ pour s'assurer leur bienveillance et leur protection. Cela lui rappelle \\ l'une des rengaines que l'on chante en été, à la course de che- \\ val, quand on consomme de grandes quantités de bière et que la \\ condition humaine est alors avantageusement comparéc au statut \\ peu réjouissant des divinités : "Même si l'on monte au royaume \\ des dieux après la mort, l'eau que les dieux reçoivent en offrande \\ chaque matin reste de l'eau. \\ Il y a pourtant un dieu que Lama Tsultrim prie chaque matin, \\ et qui exige plus cque de l'eau. Il s'agit de Kyerang Trakmé, le \\ protecteur courroucé du village. Il a été soumis par un lama au \\ XII" siècle, qui lui a fait jurer de protéger le village et de cesser de \\ dévorer hommes et bétail. Le démon a accepté ces conditions mais il \\ a aussi exigé d'être nourri de chair. Le lamal y a consenti en ćchange \\ de sa protection, et lui fait depuis une offrande quotidienne d'un \\ petit gâteau de pâte, un toma, enrichi de viande, d'os, de graisse \\ et de sang. À côté de l'autel est disposée une planche sur laquelle \\ se dressent plusieurs douzaines de ces figurines. Lama Tsultrim
}


allume quelques brindilles de genévrier pour purifier le temple et la maison, puis monte sur le toit avec une assiette contenant l'un de ces petits gâteaux. Il la lance alors en l'air en prononçant bruyamment Chö! - Tenez! - puis il redescend. Lorsqu'il entreprend un déplacement, il doit confectionner une réserve de toma pour que sa femme, Chödro, puisse faire l'offrande quotidienne, sans quoi le démon serait libéré du contrat qui l'oblige à protéger le village et ses habitants. Voilà huit cents ans que le contrat entre le fondateur du village, Trashi Gyaltsen, et le démon du lieu est respecté de génération en génération jusqu’à Lama Tsultrim, le propriétaire actuel du domaine.

Lama Tsultrim entend la voix d'un jeune villageois qui rappelle aux habitants de lâcher leurs chèvres. Il se rend au rez-de-chaussée pendant que Chödro prépare le thé du matin, et déverrouille la porte pour laisser sortir les animaux. On voit alors un flot de chèveres se déverser de chaque maison et se rejoindre pour former le troupeau, comme le feraient les affluents d'une rivière.

Dans la grange près de la maison, il prend une brassée d'herbes sèches mêlées à des brins d'armoise qu'il donne aux animaux dans l'enclos. En chemin, il passe devant un vieux noyer aussi ancien, dit-on, que le village lui-même.

On raconte que lorsque le fondateur de Lubrak s'est rendu pour la première fois dans la vallée, il a testé le site pour s'assurer que c'était un lieu de peuplement convenable. Il a planté deux aiguilles de fer dans le sol et les a recouvertes d'un petit panier. Une semaine plus tard, les aiguilles avaient donné naissance à un noyer qui avait soulevé le panier - le miracle était le signe que le lieu était bien choisi. Or, l'arbre énorme qui se dresse aujourd'hui à l'entrée du village pourrait bien avoir huit cents ans. Un jour, un dendrochronologiste allemand a voulu vérifier la légende en opérant un prélèvement sur le tronc. Lama Tsultrim et les autres villageois attendaient avec curiosité alors qu'il vissait lentement la longue sonde en spirale dans le bois. Mais la densité de l'immense circonférence de l'arbre était trop importante pour le métal, et la mèche y est restée coincée, laissant à la légende le bénéfice du doute. 
Lama Bön et maître de maison dans les marches...

Trashi Gyaltsen s'est donc marié et a installé là sa famille. Les démons qui vivaient dans les grottes en face de l'emplacement actuel du village l'ont nourri pendant trois ans, lui ont donné des champs avec les droits d'irrigation, et ont même fait les récoltes à sa place. Pas plus que son illustre prédécesseur, Lama Tsultrim n'est un moine; c'est un lama tantrique de la religion bön. Même s'il doit observer des périodes de célibat lors de ses retraites ou pendant certaines fêtes, on attend de lui qu'il se marie et perpétue sa lignée.

De sa grange, il voit le troupeau de chèvres déferler sur la colline comme un nuage passe dans le cicl. Plus personne n'est berger à plein temps de nos jours; les fils de deux maisonnées accompagnent chacun son tour les animaux jusqu'aux pâturages qui surplombent le village, où les chèvres se nourrissent d'épineux épars et de plants d'éphédra. Autrefois la communauté employait un berger - généralement un travailleur itinérant venu des collines centrales du Népal. Mais le dernier a quitté les lieux, personne ne sait vraiment pourquoi - à moins que ce ne soit à cause de l'incident du chien dans lequel Lama Tsultrim s'est retrouvé indirectement impliqué.

Le berger était logé dans une petite cabane à la périphérie du village. Il avait un chien : pas l'un de ces gigantesques mastiffs que prennent les nomades des régions montagneuses pour protéger leurs troupeaux des loups, mais un bâtard rageur qui était attaché à la porte de la cabane quand il n'était pas avec son maître et les troupcaux à l'affît des chacals en maraude. Comme un bon chien de garde, il aboyait et tirait sur sa laisse chaque fois qu'un visiteur s'annonçait. Mais parfois, il n'était pas attaché et attaquait les passants. Plusieurs personnes avaient été mordues. Un jour qu'il ćtait lâché dans le village, des enfants avaient réussi à l'attirer dans l'école avec des friandises. Le chien repu s'était endormi à l'ombre, et pendant son sommeil, les chenapans l'avaient muselé pour l'empêcher de mordre, lui avaient attaché les pattes et passé une corde au cou pour finalement le pendre à un pommier. Ils avaient ensuite décroché le cadavre et l'avaient précipité d'une falaise. Comme c'étaient des enfants pleins de ressources, l'un d'eux s'ćtait souvenu que Lama Tsultrim avait souvent besoin de crânes de chiens. 
Ils s'étaient donc faufilés dans la gorge en contrebas pour récupérer le cadavre de l'animal et couper sa tête qu'ils avaient offerte au lama. Celui-ci les avait remerciés d'une tape sur l'épaule et d'une poignée de fromage séché.

Le bouddhisme est venu de l'Inde au Tibet, où certains courants se sont mélangés aux traditions indigènes. La religion bön est influencée par le bouddhisme indien, mais la proportion des religions indigènes y est singulièrement plus importante que dans le bouddhisme. Pour les bönpos - les adeptes du bön -, la souree des enseignements est le fondateur légendaire de la religion bön, Tönpa Shenrab Mibo. Les enseignements sont répartis en neuf "véhicules" ou "voies". Les véhicules supérieurs sont constitués de pratiçues spirituelles visant la libération du eycle des naissanecs et des morts, tandis que les voies inférieures s'attachent principalement au contrôle des forces terrestres. Si les activités de la sixième voie sont réservées aux moines, toutes les autres sont du ressort de pratiquants laïes comme Lama Tsultrim. Le monde tibétain est peuplé d'une pléiade d'êtres surnaturcls. Ils sont neutres pour la plupart, et peu enclins à nous nuire pourvu qu'on les traite avec respect. Les dieux du sol, par exemple, n'ont besoin que de simples mais régulières offrandes. Ils sont, après tout - littéralement - des "propriétaires", comme le leur rappelle Lama Tsultrim pendant ses rituels de propitiation - "vous êtes les hôtes et nous sommes les invités!" - et les petites offrandes d'eau et de nourriture que nous, humains, leur faisons ne sont que le loyer que nous payons aux détenteurs invisibles de la terre sur laçuelle nous vivons. Mais les humains sont maladroits et offensent souvent ces dieux du sol en polluant l'environnement ou simplement en les dérangeant par mégarde. Les esprits expriment alors leur colère sous la forme de catastrophes naturelles et de maladies. La tâche du lama consiste à assurer prospérité, protection et santé à la communauté qu'il sert, en restaurant de bonnes relations entre les royaumes humain et divin. L'harmonic est maintenue en s'assurant que chaque catégorie d'êtres reste à sa place, car l'intrusion des uns chez les autres est source de malheurs. De même, les créatures célestes et souterraines 
Lama Bön et mâtre de maison dans les marches...

doivent être maintenues dans leurs royaumes respectifs. D'ailleurs, une énigmatique divinité, connue sous le nom de Vicille de la Terre, est chargée de maintenir bien fermée la porte de la terre. Le véhicule de son partenaire, le Vicux du Ciel est un mouton tandis que sa monture à elle est un chien. C'est pourquoi l'effigie que modc̀le Lama Tsultrim pour l'apaiser nécessite l'offrande d'un chien ou du moins un emblème canin comme le crâne de l'animal. Aussi le chien mal aimé du berger est-il à présent immortalisé, son crâne trônant au centre d'une magnificue effigic qui, au-dessus de l'entréc d'une étable, assure que la Porte de la Terre reste herméticuement close.

Mais les têtes de chien servent à d'autres choses encore. Toutes les puissances invisibles n'ont pas assez de s'occuper de leurs propres affaires : quelques-unes, très rares, se donnent beaucoup de mal pour nuire aux humains. Les plus craintes sont sans doute une classe de démons appelés bri, un terme parfois traduit par "vampire". Parmi les neuf types de vampires dénombrés par les bönpos, le plus commun est le chunghri, le "vampire des petits". Une famille dont plusicurs jeunes enfants sont morts l'un après l'autre, demandera un diagnostic à Lama Tsultrim, et s'il s'avère gu'un vampire est en cause, il entreprendra l'un de ses exorcismes les plus spectaculaires. La force vitale du vampire est transféréc dans une effigie imprimée sur du papier préalablement enduit de poison et sur lequel le lama a tracé des syllabes magiques avec du sang. Cette effigie est pliée puis insérée dans un crâne de chien que l'on enterre ensuite dans un trou triangulaire sous le lit de la mère endeuillée. La dernière fois que Lama Tsultrim a exécuté ce ritucl dans le village, c'était en 1982, pour une femme qui venait de perdre, en à peine six ans, son troisième enfant de moins de trois ans. La même année, elle accoucha d'un fils, Kemi, puis d'un autre, et enfin d'une fille. Tous les trois sont en parfaite santé, et Kemi aide fréquemment Lama Tsultrim lorscu'il rend visite à ceux qui lui commanditent des rituels domestiques.

De retour chez lui, Lama Tsultrim tombe sur Maila, le forgeron du village, qui l'attend dans le vestibule du rez-de-chaussée. Ceci en soi est inhabituel. Ne serait-ce que deux ans auparavant, un forgeron 
Une journée dans une vie, une vie dans une journée

n'aurait jamais passé le scuil de la maison. La Guerre du Peuple, qui a fait 15000 morts en dix ans, a pris fin il y a plus de cinq ans avec cependant des résultats mitigés : en premier lieu, les chefs des camps ennemis rechignent à s'engager dans un processus de réconciliation qui exposerait leurs torts respectifs. De plus, de nombreux insurgés se sentent trahis par les idéologues de leur propre parti, issus des classes supéricures et qui jouissent à présent de privilèges dont les ex-combattants sont exclus. On ne peut nier, cependant, que ce long conflit a permis d'atténuer les profondes inćgalités de caste qui caractérisaient le seul royaume hindou au monde avant sa transformation en république en 2008. Et la présence de Maila dans la demeure de Lama Tsultrim marque tout de même un pas vers une société plus inclusive. Le lama sort ses almanachs, les compulse, et déclare qu'il y aura une journée propice dans deux jours. Maila hoche la tête avec reconnaissance.

Le jeune Maila, au contraire de son défunt père, ne forge plus. Un niveau de vie plus élevé et un meilleur accès aux biens manufacturés font que les villageois achètent désormais la plupart de leurs articles en métal - pots, casseroles, ustensiles - dans l'un ou l'autre des magasins toujours plus nombreux de Jomsom, la capitale du district. Ils les rapportent aussi parfois de la plaine lointaine, où ils sont moins coûteux. Maila demeure cependant un membre important de la communauté par d'autres roies, en particulier comme métayer. Lama Tsultrim et Chödro ont laissé beaucoup de champs en jachère cette annéc, car il n'y a plus assez de bras pour aider aux récoltes. Dans le passé, la plupart des travailleurs venaient du Dolpo, une région d'une altitude plus élevée et done plus froide que le Mustang, à plusieurs jours de marche à l'ouest. La saison agricole $y$ finissant plus tôt, les migrants hivernaux en route vers le sud gagnaient facilement de l'argent comme moissonneurs ou batteurs dans les terres plus basses. Mais entre-temps les gens du Dolpo ont cessé de proposer leurs scrvices car ils se sont enrichis d'un coup grâce à une ressource naturelle qui pousse en abondance dans les prairies d'altitude : le yaitsa gumbu, "herbe en été, ver en hiver ", la célèbre chenille-champignon, atteint sur le marché chinois 
Lama Bön et mâtre de maison dans les marches...

en plein essor des sommes fabuleuses. Maila est donc très demandé, et Lama Tsultrim l'a retenu longtemps à l'avance.

Cela va être une journée particulièrement chargée parce que son ami anglais, l'anthropologue, veut qu'il fasse une propitiation des esprits serpent. Les chartes astrologiques du lama indiquent que c'est un jour propice - un thep - ct le rituel ne sera donc pas une simple mise en scène mais aura un récl bienfait pour le village. Pourtant, le lama objecte que le temps manque déjà pour le battage du sarrasin. L'anthropologue délègue aussitôt son filleul qui l'a accompagné, auprès du lama pour aider à la tâche. Le filleul ne parle pas tibétain, et se demande pourquoi Lama Tsultrim lui sourit avec reconnaissance.

Pourtant Lama Tsultrim se montre encore réticent à effectuer le rituel aujourd'hui. Si les esprits serpents sont satisfaits des offrandes, ils vont faire pleuvoir, et cela mécontentera les villageois qui, cux, ont besoin que le sarrasin récolté sèche bien dans les champs avant de le rentrer. L'ami anglais ne répond pas à cette objection, mais présente un visage secpticue en regardant le ciel d'octobre sans nuage.

Aussitôt après avoir prié ses dieux domestiques, Lama Tsultrim se rend sur le plus haut toit de sa maison pour appeler se's enfants à New York avec son portable. La couverture du réseau téléphonique s'est récemment améliorée, et il n'a plus besoin de marcher une heure jusqu'en haut de la crête, en face du village, pour capter le réseau. Son plus jeune fils, Pema, s'est marié et vient d'avoir une petite fille. Il assure son père que la famille, la soeur de Pema et son grand frère, Rig/in Angyal, vont tous bien. Mais lorsque Lama Tsultrim redescend sur le toit inférieur de la maison, qui sert de terrasse, il est perdu dans ses pensécs. Il s’interroge sur l'avenir Ae son fils aîné, Rigdzin Angyal. d'en bas. "Grand-père Tsultrim ! Vous êtes là ?" Encouragée par la réponse affirmative du lama, la jeune Nangdza grimpe sur la grossière échelle de bois qui mène jusqu'au toit, où Tsultrim est assis à l'ombre d'un auvent. Elle s'approche de lui avec un sourire timide

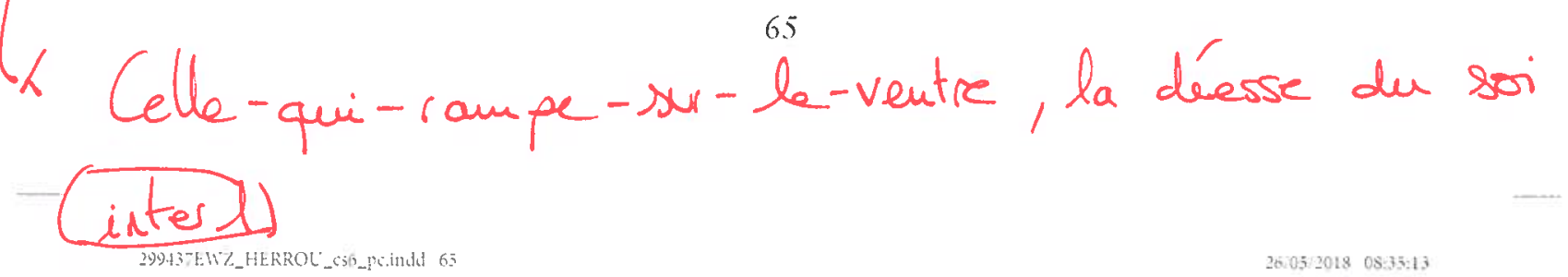


Une journée dans une vie, une vie dans une journée

et pose sur la table devant lui une bouteille remplie d'alcool d'orge, arak, qu'elle a clle-même distillé puis, à côté, une écharpe blanche. Ces écharpes sont l'objet rituel le plus utilisé entre Tibétains. Elles n'ont pas de valeur matérielle (outre le coût minime du tissu), mais elles sont, avec les gestes qui les manipulent et les circonstances dans lesquelles elles sont offertes ou échangées, un vecteur omniprésent de messages. L'offrande d'une écharpe peut exprimer une félicitation, une bénédiction, l'humilité, la demande d'un enseignement spirituel ou encore la requête d'un service - tout dépend de la façon dont l'écharpe est à la fois offerte et reçue, et parfois aussi des objets qui accompagnent le geste. Lorsque l'écharpe est pliée en deux et posée sur une table à côté d'une bouteille d'alcool, comme à présent, il s'agit d'une requête solennelle.

Nangdza explique qu'elle et son mari, Samdrup, aimeraient construire une grange au bord de l'une des aires de battage, ce qui implique de creuser le sol. Nangdza et Samdrup prévoyaiene de construire leur grange dans deux mois seulement, après les récoltes, lorsqu'il y aura de la main-d'oeuvre disponible, mais comme Lama Tsultrim passe parfois l'hiver au sud, il vaut micux le consulter tant qu'il est là. On ne creuse pas le sol n'importe comment; les dieux qui $y$ habitent pourraient prendre ombrage d'un manque de respect à leur égard, et se retourner alors vers ceux cui les auraient offensés - et vers toute la communauté - en déclenchant des tempêtes de grêle ou des maladies. Surtout, lorsyue l'on ouvre la terre, il faut être très attentif à ne pas toucher Toché, Celle qui Rampe sur le Ventre, n'importe où. La position dans lacuelle la déesse-serpent est allongée varie dans l'année, mais un almanach ordinaire ne peut nous renseigner sur sa position, aussi Lama Tsultrim doit-il consulter un certain manuel de géomancie.

Il pénètre dans le temple domestique contigu au toit-terrasse de sa maison et se dirige vers une étagère sur laquelle sont entassées des liasses oblongues enroulées dans une pièce de tissu - sa bibliothèque, un trésor de sagesse enveloppé dans des chiffons déteints. Là se trouvent les invocations aux dieux du panthéon bön, des plus grands bouddhas juscu'aux humbles protecteurs de la terre; 
Lama Bön et mâtre de maison dans les marches...

des ritucls pour attircr la chance et la prospérité, exorciser démons et sorcières, récupérer les âmes errantes, traiter les victimes d'attaques cérébrales par des mantras thérapeutiques, consacrer les temples, libérer les malades de l'emprise d'esprits prédateurs ou encore faire des offrandes au feu. On peut appeler Lama Tsultrim à tout moment pour faire l'un ou l'autre de ces rituels, certains doivent être exécutés à intervalles réguliers, le plus courant étant la propitiation des dieux domestiques. Le lama a près d'une cinquantaine de patrons à qui il rend visite chaque année dans le district népalais du Mustang. La plupart se disent bouddhistes mais leurs dieux domestiques appartiennent à la religion bön comme Absé la Lance Rouge, Absé Dungmar, le chef des protecteurs effrayants nommés tsen. Parfois, la divinité est une déesse protéiforme, généralement connue sous le nom de Sipé Giyalmo, la Reine de l'Univers. Le dieu domestique de Lama Tsultrim est le roi blanc Nyipangsé, un souverain divinisé de l'ancien royaume de Zhangzhung. Le Mustang aurait fait partic de ce royaume mystérieux avant cju'il ne soit ćclipsé par l'Empire tibétain au vir' siècle.

Lama Tsultrim sort du temple avec un long paquet enveloppé d'une pièce de tissu qu'il déballe pour révéler une demi-douzane de manuscrits rectangulaires. Certains sont des feuilles volantes tandis que d'autres sont constitués de folios reliés par le haut pour les maintenir en ordre, mais tous sont usés et tachés par de nombreuses années de consultations répétées. Le lama extrait l'un des textes de son enveloppe de tissu, et cherche avec dextérité dans les folios jusqu'à ce qu'il trouve un curieux outil en relief fixé dans la marge de gauche du manuscrit : deux disques de papier, le plus petit posé sur le plus grand, reliés à la page par une mèche de papier qui sert de pivot. Le bord extérieur du disque inféricur est divisé en douze segments, contenant chacun unc ou deux syllabes, tandis que le disque supérieur représente une créature avec un torse humain surmonté d'une tête de bête à cornes. La partic basse de son corps s'amincit en une queue à écailles, ne laissant aucun doute sur l'identité de la déesse Toché, qui réside sous la terre. 
Une journée dans une vie, une vie dans une journée

Sa position change dans l'année à mesure qu'elle tourne lentement sur elle-même au fil des mois, et il faut faire attention de ne pas la frapper au mauvais endroit. Le texte accompagnant le diagramme est très explicite à ce propos : "Si elle est frappée à la tête, l'homme, ses parents et ses enfants mourront, et ce sera particulièrement néfaste pour le prêtre. Il en va de même si elle est frappée sur la crinière ou sur le visage. [... Si elle est frappée à la queue, il y aura des conflits, et ce sera mauvais pour les troupeaux. Mais si vous creusez sur son ventre, ce ne seront que bienfaits et bénédictions. Si vous creusez sur son dos, ce ne sera pas bon pour vous - les ceurs de vos jeunes frères et sceurs éclateront, et vos cnfants mourront. "

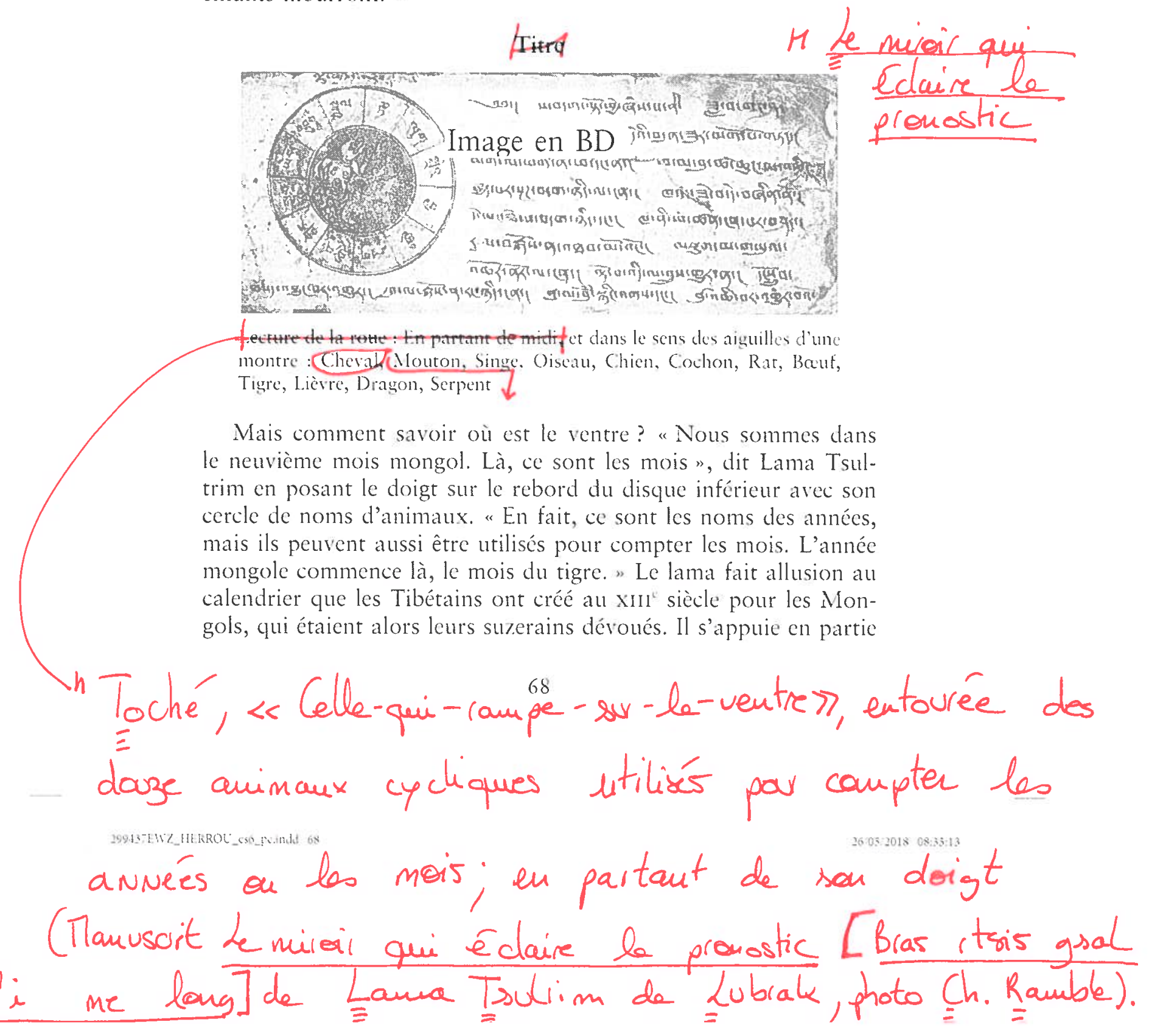


Lama Bön et maître de maison dans les marches...

sur le système spirituel du bouddhisme indien connu sous le nom de Kalachakra, la Roue du Temps, sclon lequel le cycle annuel commence au printemps. La méthode de comptage des années, qui se réfère à une série d'animaux de douze ans, existe aussi en Chine, mais les Tibétains l'ont adoptée depuis sa plus ancienne source centrasiatique. Comme le calendricr "mongol » est celui qu'utilise la communauté tibétaine en exil dans ses almanachs annuels, e'est aussi le système qu'utilise généralement Lama Tsultrim pour ses calculs. Il lit à haute voix les premières lignes du texte géomantique : "Quel que soit le mois où cela tombe, c'est là qu'il faut placer sa queue. Sa tête touche le "septième marginal" " - d'après les explications du lama, on commence à partir de n'importe quelle case et on en compte sept dans l'une ou l'autre direction pour trouver le "septième marginal ". Les animaux qui se font face représentent des attributs mutuellement incompatibles. Par exemple, un homme et une femme nés respectivement en 1986 et 1993 (années du liève et de l'oiseau, a sept ans l'écart) ne devraient pas être autorisés à se marier; ou alors, s’ils y sont déterminés et que leurs familles sont consentantes, il faudra cu'ils commanditent l'exécution de certains rituels pour écarter les dangers qu'entrainerait leur incompatibilité astrologicjue.

À la perpendiculaire de la ligne qui va de la tête à la queue de Toché - c'est-à-dire dans les cases du serpent et du cochon respectivement - se trouve le singe. Mais quelle est la direction cardinale représentée par le singe? La réponse ne se trouve pas dans le diagramme, ni d'ailleurs nulle part dans ce texte géomantique. C'est ce qu'un spécialiste comme Lama Tsultrim doit apprendre au cours de sa formation : la création de l'univers à partir du corps de la tortue cosmique. D'après une ancienne ocuvre cosmogonique de la tradition bön, l'univers a été créé par une divinité nommée Künbum Gojé, Celui qui Répartit l'Espace pour Toutes les Centaines de Milliers. Künbum Gojé " a rassemblé le lustre de l'un de ses cheveux, la salive de sa bouche, et l'éclat métallique de ses ongles, la chaleur de son corps et le souffle de sa bouche, les impuretés à la surface de son corps et la radiance de son esprit. Puis, à l'aide de ces 
ingrédients, il a écrit les Neuf Syllabes Hérö̈ques des éléments sur une précieuse tablette en or avant de la jeter sur le sol. Du centre de la terre dorée apparut alors la fondation des vies du monde : une tortue jaune en or" .

Les parties du corps de la tortue engendrèrent les cinq éléments, et ceux-ci furent associés à l'une ou l'autre des directions cardinales, à des animaux, des trigrammes, des étoiles et des planètes. Les poumons de la tortue produisirent l'élément fer, disposé à l'ouest et associé au singe, à l'oiscau, à la planète Vénus, et enfin au trigramme $=$ appelé dwa en tibćtain (ch. dui).

Le singe, done, désigne l'ouest. Samdrup est encore un jeune homme, et Lama Tsultrim veut être sûr qu'il sait comment ouvrir le sol lorsque commenceront les travaux pour la grange. "L'ouest, c'est par là ", dit-il, comme si Samdrup ne le savait pas déjà. "Quand tu creuses, mets-toi comme ça. " Il est debout, les jambes écartées, face à l'ouest, et fait semblant de creuser. "Comme ça, tu toucheras son ventre. Et bien sûr ", ajoute t-il, " quand tu casses la première motte de terre, il faut commencer avec une corne d'antilope, pas une pioche en métal. Tu en as une?". Samdrup l'assure qu'une corne d'antilope est attachée au pilier central de sa maison, comme dans la plupart des maisons du village, avec les anciennes armes que les dieux martiaux de la maison affectionnent particulièrement. Il n'y a pas d'antilope dans l'Himalaya, mais peut-être yue les cornes de ces animaux en voie de disparition sur le haut plateau furent les outils employés par les premiers Tibétains pour creuser le sol - et si ect ustensile était dans le contrat d'origine passé avec les susceptibles dieux de la terre, autant ne pas les provoquer en innovant.

A Après le départ de Nangdza, Lama Tsultrim retourne dans le temple pour rassembler les accessoires nécessaires à la propitiation des $l u$, les esprits serpent : le tambour portable à long manche et la cloche plate sont utilisés dans beaucoup de cérémonies, mais il saisit aussi tout l'attirail qu'il n'utilise que pour le culte de ces étranges êtres souterrains : platcaux, bols, cuillères et supports en cuivre - le seul métal accepté par les lu; des cartes peintes représentant
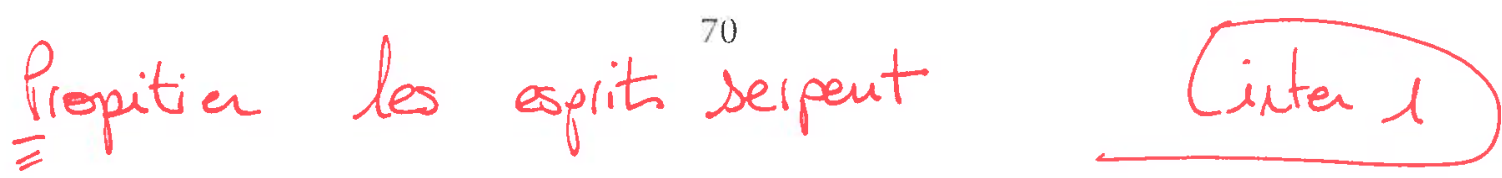
Lama Bön et mâtre de maison dans les marches...

un groupe de ces esprits du sol; du lait et des herbes médicinales pour les nourrir car au contraire de nombreuses divinités sauvages, les lu évitent la viande et l'alcool ; et bien sûr les textes dédiés au culte de ces créatures.

Le chemin monte droit sur le versant de la colline, au sud du hameau, et passe à côté du petit temple construit autour de la grotte où, dit-on, le fondateur du village a médité pendant neuf ans. Neuf encoches apparues spontanément sur le rocher qui surplombe la grotte, témoigneraient chacune d'une année de sa retraite. Dans le temple se trouve encore une grosse pierre portant l'empreinte de pied du lama. Et les rochers à l'entour portent d'autres marques étranges, des traces de sabots des chevaux et autres ongulés qui ont visité la grotte. Le grand nombre de ees pétroglyphes suggère que ce lieu était un site de culte important d'une religion qui aurait précédé le bön. En regardant vers le bas de la colline de l'autre côté de la gorge, Lama Tsultrim peut discerner des grottes dans la falaise orientée au sud et faisant face au village. Ce complexe de grottes préhistoriques, l'un des milliers que comptent les vallées du Mustang, a été abandonné depuis des siècles, mais deux grottes sont encore utilisées par les lamas pour leurs retraites. Lama Tsultrim voit encore l'ocre rouge de l'enduit à l'entrée de la grotte où il a passé de nombreux mois de solitude pour apprendre à méditer et perfectionner sa connaissance des rituels que lui avait appris son père. C'est là aussi qu'il a installé son fils et héritier spirituel, Rigdzin Angyal, pour qu'à son tour il pratique ce qu'il lui a enseigné.

Après une heure et demie de marche, le lama et ses compagnons parviennent à leur destination, une source cristalline entourée d'immenses genévriers, la résidence principale des esprits serpent de Lubrak. Lama Tsultrim s'assied sous l'abri naturel formé par le tronc fendu de l'un de ces arbres et installe un autel portable entre lui et le cours d'eau. L'autel consiste en une petite table basse couverte d'une pièce de tissu sur laquelle il dispose les objets du culte : pots en cuivre remplis d'eau et de lait parsemé de pétales de calendula, des petits cônes et des boules de pâte d'orge - la nourriture et la boisson des esprits -, un vase fait de feuilles provenant de la 
forêt et, juste devant lui, le long livre de feuilles libres dans lequel se trouve l'invocation qu'il va chanter. Le tintement de la cloche plate annonce le début du rituel, et bientôt le bourdonnement de la litanie, puis le grondement léger du tambour, font venir les esprits serpents de sous les arbres sombres pour recevoir leurs offrandes.

Après le rituel, Lama Tsultrim propose de marcher jusqu'à lá crête voisine d'où l'on embrasse du regard la vallée de la Kalli Grandaki. C'est peut-être la plus profonde gorge du monde et, jusqu'aux années soixante, l'une des artères commerciales les plus empruntées entre le plateau tibétain et les plaines indiennes. Luttant contre les rafales de vent, lui et ses compagnons peuvent aperevoir Jomsom au sud; vers l'ouest, les villages de Pelag et Dangkardzong; au nord-est, la piste mène vers une autre crête avant de passer dans la vallée de Muktinath. Les collines et les villages baignent dans le soleil d'automne. L'ami anglais est content car il a pu filmer les deux heures de rituel - sauf les dix minutes de la partic la plus importante de la cérémonie, pendant lescuelles la caméra et l'enregistreur audio ont tous deux cessé de fonctionner. Mais il ne s'en fait pas trop pour ça, parce qu'il sait que c'est quasiment la règle dans les recherches sur le terrain. Ce qui lui parait beaucoup plus irritant est que, dans le ciel bleu immaculé, un nuage se forme, un seul, juste au-dessus de Lubrak.

Lama Tsultrim suggère que la petite troupe s'abrite du vent pour déjeuner. De retour dans le bois de genévrier, il demande à son assistant de faire un feu, et il mélange de la farine d'orge avec de l'eau de source dans une marmite en fer (forgée par le père de Maila). Cette bouillie épaisse qui tient au ventre constitue l'aliment de base des villageois. À la façon des bergers, il ramasse de larges écorces laissées par la mue des boulcaux et les distribue à ses compagnons en guise d'assiettes. À la fin du repas, les premières gouttes commencent à tomber.

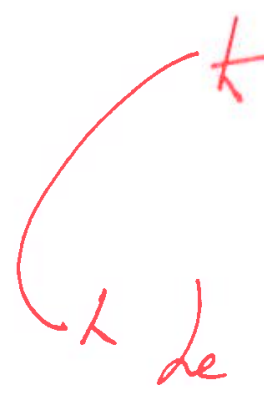

A peine de retour au village, Lama Tsultrim rencontre un messager de Tiri venu l'inviter à faire un ritucl de déménagement. La nouvelle maison est construite, mais l'autel domestique n'a pas encore été transféré. Le lama accepte d'un air las de s'y rendre
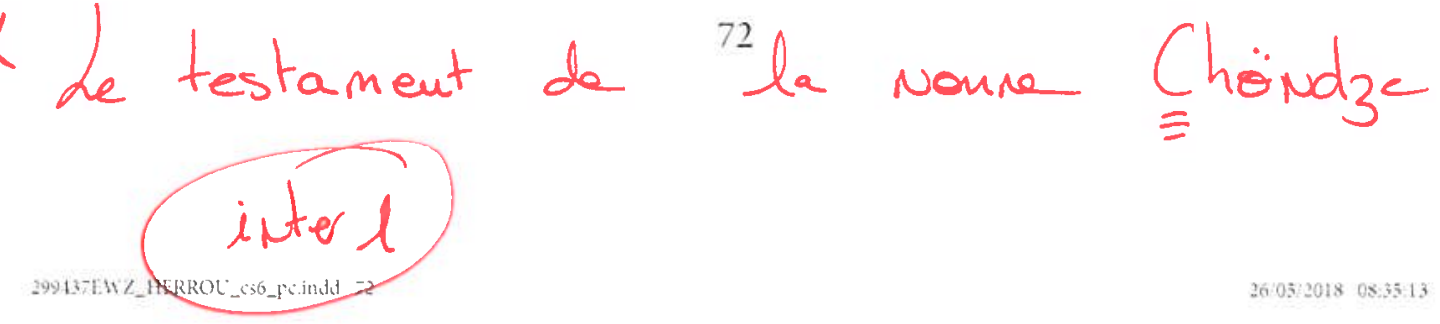

Nyishar 
Lama Bön et maître de maison dans les marches...

pour s'en occuper. Kemi l'aidera, mais ce serait tellement micux si Rigdzin Angyal était là pour prendre les choses en main.

Lama Tsultrim entend un cri sur le toit d'un voisin : quelqu'un arrive de la vallée. Il monte sur le toit et distingue deux silhouettes qui marchent vers le village. Sans pouvoir encore les distinguer, il devine qu'il s'agit de sa sour Namgang et de son beau-frère, Pema Dorjé. Pema Dorjé est un homme très riche qui vit dans une grande maison à Katmandou. Autrefois il n'aurait pas fait un pas en marchant, il était toujours à cheval. Mais de nos jours, on ne voit plus de chevaux sur les chemins. La plupart ont été vendus depuis qu'une piste carrossable relie Jomson, où se trouve le petit aćroport, à Muktinath. Tous ceux qui viennent à Lubrak doivent à présent ou bien marcher deux heures ou bien prendre une Jeep publique jusqu'à la confluence de la Pandak Khola et de la Kali Gandaki, puis remonter en marchant une demi-heure vers l'est, le long de la Pandak jusclu’à Lubrak.

Tsultrim reçoit chaleureusement sa scur et son beau-frère, pour lequel il éprouve une sincère affection. Malgré sa grande fortune, Pema Dorjé n'est pas devenu arrogant comme le sont parfois les villageois enrichis, et il ne méprise pas ceux qui sont restés au village. Il possède encore des terres dans la vallée de Muktinarh au nord de Lubrak, auxquelles la présence de la route a donné beaucoup de valeur. Sa visite est motivée par l'acte de vente d'une pareelle près de Muktinath qu'il vient de céder à Lama Tsultrim à un prix très raisonnable. Il est venu pour apporter l'acte de transfert que lui et Tsultrim ont signé deux jours plus tôt au bureau du cadastre. Chodrö s'affaire pour apporter des tapis et des tables basses sur la partie couverte de la terrasse. Elle propose un pichet d'arak, Tsultrim en accepte volontiers une tasse mais Pena Dorjé refuse. Incapable de boire modérément, il préfère ne pas boire du tout.

Pema Dorjé tend l'acte au lama, qui retourne une fois encore dans le temple. Il ressort avec un cylindre métallique d'où il retire un sac en plastique rempli de papicrs. Contrairement aux longs folios volants enveloppés de tissu, ces feuilles d'un papier plus léger, sont soigneusement repliées sur elles-mêmes segment par segment 
Une journée dans une vie, une vie dans une journée

et peuvent atteindre plusieurs mètres de long. Y sont enregistrés des actes de propriété, des contrats de prêt, des reçus fiscaux ou autres rapports domestiques. Chaque maisonnée possède de ces documents qui recèlent une partie de l'histoire familiale sur plusieurs générations.

L'ami anglais les connaît bien, ils sont un élément important de l'histoire du village. Mais il y en a un cu'il rêverait de lire, un document d'une importance cruciale pour l'histoire de la famille, mais que le lama a toujours dit avoir égaré. L'ami lui demande poliment pour la centième fois, sans guère d'espoir, s'il l'aurait par chance retrouvé. Après une courte hésitation, le lama répond qu'effectivement, il est tombé dessus récemment. Son ami souhaiterait-il y jeter un coup d'xil ? Volontiers.

Tout le monde aime les histoires de famille, aussi Pema Dorjé et Namgang s'assoient-ils confortablement pour écouter Tsultrim. Le lama déroule le long document et commence à le lire, en faisant régulièrement des pauses pour ajouter des commentaires. L'assistance connât les grandes lignes de l'histoire, bien sûr, mais elle n'a jamais été racontée de façon si détaillée. Le document est le testament de la nonne Chöndzé Nyishar, le dernier membre du clan Yangal fondateur de Lubrak. Ses dernières volontés ont été consignées en 1872, alors qu'elle était dans sa quatre-vingt-septième annéc.

Le mot tibétain pour clan désigne littéralement "l'os". Nous héritons nos os de notre père, et notre chair de notre mère. Un clan est constitué d'individus qui partagent le même os, et le membre le plus âgé du clan ou d'un lignage au sein de ce clan est appelé dungdzin, "détenteur de l'os". Même si Chöndzé Nyishar n'avait pas été nonne, clle n'aurait jamais pu transmettre l'os du clan Yangal à l'un de ses enfants.

Le testament explique comment, après la mort des trois fils de son frère aîné, elle a cherché des héritiers dans l'enclave voisine du Dolpo, où prospérait le clan Yangal (comme encore de nos jours). Elle a bien trouvé un jeune homme dans le village de Bicher, mais il est mort peu après avoir emménagé à Lubrak. D'autres lui ont 
Lama Bön et mâtre de maison dans les marches...

succédé, mais eux aussi sont morts sans descendant. L'opportunité d'hériter d'une maison prestigicuse dans la vallée relativement douce de Lubrak (à une altitude de seulement 3000 mètres) aurait pu être une perspective séduisante pour quiconque habite les rudes régions montagneuses du Dolpo. Pourtant après plusicurs tentatives malheureuses, les Yangal de cette région ont hésité à envoyer leurs fils dans ce qui était en train de devenir un cimetière de leur clan. Après ce long préambule, le testament déclare que Chöndzé Nyishar lègue le domaine à un jeune homme de Lubrak d'ascendance inconnue, Pema Samdrup (murmures d'approbation de l'auditoire captivé). Bien qu'il ne possède par l'os Yangal, Pema Samdrup est donc devenu le détenteur du domaine, héritant ainsi des propriétés, des obligations et de son réseau de patrons. Cinc générations plus tard, le détenteur de l'os, et du domaine, est Lama Tsultrim.

La lecture de l'acte fournit l'occasion d'évocpuer un problème devenu de plus en plus pressant ces demières années. Et tandis que l'ami anglais accepte avee gratitude l'offe du lama de photographier le document, l'assistance évoque un sujet tabou : qui sera le successeur de Lama Tsultrim?

LLe dernier
lama?

K Les lamas de la maison Yangal de Lubrak assurent le service rituel de leurs patrons en ligne ininterrompue depuis plus de huit siècles. Même lorsque la lignée fondatrice s'est éteinte, une nouvelle famille - l'ancêtre de Lama Tsultrim - a maintenu la tradition. Les habitants du Mustang, y compris les lamas de Lubrak, ont toujours fait du commerce à longue distance, se rendant en Inde et au-delà en automne, après les travaux des champs. Mais il fallait revenir avant le Nouvel An, en février. C'était la règle. Aujourd'hui, presque un dixième de la population du pays vit à l'étranger d'où les travailleurs envoient régulièrement de l'argent à leur famille, sans quoi les villageois pourraient difficilement survive en pratiquant une agriculture de subsistance. Pour les habitants du Mustang, les États-Unis sont une destination privilégiée. C'est parce que trois des quatre enfants de Lama Tsultrim sont à New York qu'il a pu acheter des terres à Muktinath. Une de ses deux filles, Sangyé, est propriétaire de l'hôtel Bob Marley, très fréquenté par les trekkeurs occidentaux 
Une journée dans une vie, une vie dans une journée

sur le circuit de l'Annapurna. Mais cela fait maintenant plus de dix ans que Rigdzin Angyal, son fils aîné, réside à New York, ct tous s'accordent à dire qu'il est vraiment temps qu'il revienne prendre en charge le domaine. Pourtant c'est bien Pema Dorjé qui a facilité le départ de Rigdzin Angyal aux États-Unis. En effet, même si l'immense majorité des demandes de visas par les citoyens népalais échouent, en payant l'équivalent de 10000 dollars à Pema Dorjé, l'aspirant-migrant trouve sa demande de visa acceptée. Une fois en Américue, il trouvera un travail lucratif dans la construction ou la restauration ou, si c'est une femme, comme assistante maternelle ou dans un salon de beauté. Après le remboursement de la dette contractéc pour partir, il commenecra à envoyer de l'argent au pays. Rigdzin Angyal a particulièrement bien réussi, et il est maintenant chef sushi dans un restaurant japonais de Manhattan. Il sait bien qu'il est temps pour lui de reprendre les devoirs sacerdotaux de son père. Tagla, l'autre lama bönpo au sud du Mustang, un parent de Tsultrim, est mort l'an dernier. L'un de ses fils est moine en Inde tandis que l'autre n'a jamais suivi d'enseignement pour être lama. Il est probable que, se retrouvant sans prêtre, le réseau de clients de Tagla, abandomne le bön pour se tourner vers le clergé bouddhiste. Lama Tsultrim tripote son portable, puis le remet dans un pli de sa robe. Tout le monde dort à New York à cette heure.

Les champs ont séché après l'averse du milieu de matinée, et il est temps d'aller battre les derniers tas de sarrasin. Avec l'aide de Maila et du filleul de son ami anglais, le travail est vite fait. Les batteurs sont encouragés par de grandes quantités de bière. Lama Tsultrim fait une pause et s'assoit au bord d'un champ. Mais la longue marche matinale, l'arak au soleil et maintenant la bière font qu'il s'endort aussitôt. Les batteurs se moquent gentiment de lui : "Alors lama! Et votre méditation? Vous avez des visions?"

Les lamas tantriques héréditaires comme Tsultrim ne sont pas des moines célibataires ni même des crmites laïques. Ils vivent dans le monde, se marient et ont des enfants - des fils - pour perpétuer la lignce sacerdotale. Les chefs des quatorze maisons de Lubrak sont, comme le veut la tradition, des lamas héréditaires mais personne 
Lama Bön et maitre de maison dans les marches...

dans la région n'a une ascendance aussi prestigieuse et un cercle aussi vaste de patrons que Lama Tsultrim. Comme dans la plupart du monde tibétain, la "maison " - qu'il faut considérer comme un domaine - est une entité sociale aussi importante que complexe. Elle est typiquement constituée d'un groupe de frères mariés à une seule femme, une configuration qu'on appelle polyandrie fraternelle. La maison a certaines obligations envers la communauté, par exemple fournir le personnel pour le service public ou la main-d'ouvre pour les travaux publics et le paiement des taxes villageoises et en retour a des droits sur les ressources communales telles que forêt, pâturages et surtout irrigation. Une maison comprendra la demeure principale et les champs; souvent aussi des maisons secondaires habitées par exemple par des parents âgés ou des socurs non mariées. Ces résidences ne feront pas l'objet d'héritages séparés après la mort de leurs occupants mais retourneront à la maison mère. Les frères cadets peuvent parfois devenir moines, et bénéficier de l'éducation religieuse qu'offrent les monastères établis par les réfugiés tibétains en Inde et au Népal. Ainsi, Tshultrim a un frère cadet, Trashi, cui a passé plusieurs années comme novice en Inde avant de revenir au village en 1982 et de reprendre sa vie de laïc. Savoir si Trashi devait se joindre à la famille de son frère ainné ou ćtablir sa propre famille a été l'objet de nombreux débats. La polyandrie est de moins en moins praticuée dans les hautes terres de l'Himalaya, en partie à cause des règles d'héritage qui ont changé et de certains facteurs ćconomiques, mais en partic aussi à cause d'une sensibilité culturelle non tibétaine venue du Sud, qui fait de la polyandrie une coutume exoticue. Toutes ces raisons, en plus du fait que la maison de Tshultrim était de belle taille, comprenant deux grandes demeures et de nombreux champs, ont fait que Trashi s'est installé séparément, opérant ainsi une première division.

Le plus gros de l'activité religieuse de Tshultrim consiste à accompagner les autres lamas de la communauté dans les cérémonies collectives qui se tiennent périodiquement dans le temple du village, ou bien à exécuter des ritucls pour son réseau de patrons privés. Certains lamas entreprennent des retraites pour méditer dans leur 
Une journée dans une vie, une vie dans une journée

autel domestique ou dans les grottes près du village, exercices ésotériques visant à développer une réalisation spirituelle. Certaines de ces retraites impliquent de se laisser cmmurer dans une grotte et d'y rester dans l'obscurité la plus complète pendant 49 jours. Lama Tshultrim parle parfois d'entreprendre une de ces retraites mais entre ses travaux domestiques et ses clients qui le sollicitent sans cesse, il n'en a jamais le temps.

Le sarrasin est emballé dans des sacs, cousus avec des ficelles et transportés dans la grange près de la maison. Une silhouette grimpe sur le toit du temple et crie dans la vallée pour convoguer une réunion de village.

Le temple, un bâtiment construit autour de six piliers datant du XIX" siècle, accueille la vingtaine de cérémonies annuelles exécutées par les lamas. Personne n'y vit, même si pour l'exorcisme du village les lamas doivent dormir dans le périmètre du temple; aussi, un intendant, une fonction que chaque maison remplit par rotation annuclle - vient faire des offrandes d'eau chaque matin et allumer une lampe à beurre chaque soir. Mais le temple sert aussi de salle communale et la réunion annoncée n'est pas religicuse.

Lama Tsultrim sait qu'il n'est pas pressé et çu'il peut finir de rentrer la récolte avant de répondre à l'appel. Autrefois - il n'y a ne serait-ce qu'une décennic -, les réunions étaient annoncées par le chef de village du haut de sa maison, au son de son tambour. Il commençait par un battement sourd puis accélérait progressivement le tempo pendant dix minutes donnant ainsi un sentiment d'urgence. Quiconque n'était pas arrivé à la fin de l'appel devait payer une amende pour son retard. Maintenant la règle n'est plus suivic, on ne bat plus le tambour et tout le monde arrive en retard. Le temple sert de lieu de réunion pour le village et pour les cérémonies de la communauté des lamas - traditionnellement un ou deux hommes par maison. Lorsque finalement Lama Tsultrim grimpe jusqu'au temple, quelques personnes sont déjà là. Les masques et les robes utilisés pour les danses annuelles pendent aux chevrons pour les protéger des souris, mais l'autel est vide. Il se souvient de l'époque où deux pleines rangées de lamas accomplissaient les cérémonics 
régulières. Les aînés sont morts, et les jeunes ont émigré aux ÉtatsUnis, ou bien, se sentant laissés pour compte et démoralisés, ils s'élèvent contre l'autorité traditionnelle des anciens. La plupart de ces jeunes hommes ne connaissent pas le tibétain. Cependant, quand bien même ils ne peuvent lire les textes, ils restent des lamas. La tradition exige d'eux qu'ils prennent part aux cérémonies, dans lesquelles ils occupent surtout des tâches subalternes, comme servir le thé ou la bière et faire les offrandes.

Cette année, le Dögyab - la principale cérémonic annuelle - a mal tourné. Les lamas sont censés faire une retraite, comme l'indique le petit cairn de pierres rouges construit à la porte du temple pendant cette période. Ils doivent dormir dans le temple et éviter tout contakt intime avec une femme. Cette fois, les jeunes ont dormi dans la salle de réunion au-dessus du temple, où ils ont fumé et, d'après le précepteur, se sont comportés de manière inappropriée. De plus, pendant la cérémonie, lorscue les lamas plus âgés leur ont donné des instructions sur ce qu'il fallait faire dans le temple, ils ont répondu de manière agressive. L'un d'cux a même frappé l'un des anciens.

Lama Tsultrim se souvient qu'autrefois, ses acolytes et lui n'étaient pas très différents des jeunes d'aujourd'hui, mais ils craignaient l'autorité de leurs aînés. Quand les vieux lamas dormaient, les jeunes escaladaient les fenêtres en utilisant la ceinture de leur robe conme corde et partaient furtivement rejoindre leur femme ou leur petite amic. Ils attendaient ensuite près de la porte du temple que les vieux yui avaient bu trop de bière sortent au petit matin pour se soulager. Ils se glissaient alors à l'intérieur sans être vus. Mais s'ils étaient pris, ils étaient punis. Les coupables avaient le choix entre l'humiliation de se déshabiller et de faire, nus, des prosternations dans le temple devant les autres lamas, ou d'être flagellés avec l'un des fouets pendus à un pilier du temple. Lama Tsultrim se souvient qu'il avait lui-même été pris et qu'on lui avait donné le choix. Il avait opté pour les prosternations.

Les jeunes sont les derniers à arriver. La réunion porte sur l'organisation du foyer, une institution crééc à la reçuête des villageois par un sponsor privé il y a une dizaine d'années. L'école publique 
Une journée dans une vie, une vie dans une journée

du village, contrairement aux écoles gouvernementales, dispense un enseignement en tibétain, ainsi que des cours supplémentaires de science et d'anglais. Bien qu'il s'agisse d'une école lä́que, des fonds sont aussi prévus pour une instruction religieusc bön, absente de toutes les autres écoles tibétaines privées dans le pays. Le foyer attire une centaine de pensionnaires des villages alentour. Les quatorze maisons du village sont représentées par une personne dans le conseil. Mais les ainés et les plus jeunes s'affrontent. La réunion est tenduc. Il est évident qu'ils ne vont pas s'entendre, et que l'école en pâtira. Lama Tsultrim suggère que la moitié du conscil soit constitué de femmes: "Une partic femme et une partic homme", dit-il. Mais le terme qu'il utilise pour "partie" est gyacha, "pourcent". Un pourcent homme, un pourcent femme. Les chiffres n'ont jamais été son fort. Pourtant même cette solution ne s'avère pas satisfaisante. Les femmes forment un groupe uni, mais elles parlent peu dans les réunions de village, car même si les temps changent et que leur statut s'améliore, les hommes peuvent encore les huer juste paree qu'elles sont des femmes.

Puis quelqu'un suggère que l'entière gestion de l'école soit laissée aux femmes. Lama Tsultrim n'arait jamais pensé à cela, mais l'idée lui plait et il la soutient immédiatement.

Jusqu'à récemment, la communauté comptait encore un petit nombre de nonnes qui étaient cependant très dynamiques, mais la dernière, une femme d'au moins 80 ans, est morte il y a quelques années et la tradition est tombée en désuétude. Les nonnes n'utilisaient pas le temple mais se rassemblaient au sein de leur maison de famille pour réciter des prières sous la direction de Lama Tshultrim. A part leurs cheveux coupés, peu de chose les distinguent des villageoises ordinaires et elles avaient peu d'éducation mais leur dévotion et leur engagement étaient tout à fait réels et Lama Tshultrim était désolé de voir la tradition s'éteindre. Le groupe des femmes mariées a été de plus en plus actif au cours des dernières années et bien qu'il s'agisse d'un groupe séculier, leur importance grandissante est quelque chose que Lama Tsultrim voit comme un développement très encourageant. 
Lama Bön et maitre de maison dans les marches...

Elles boivent et se battent moins que les hommes, ct bien qu'elles manquent encore de confiance, la conscience croissante de leur force collective a fortement contribué à la réalisation de certains projets. Après encore quelques disputes et discussions, le conseil se met d'accord. Après le Nouvel An en février prochain, la gestion de l'école sera remise au groupe des femmes.

Le tintement métallique des cloches et les roulements de cailloux annoncent les chèvres qui rentrent des collines. Elles seront bientôt à la porte de leur enclos de pierre. La réunion s'achève. Lama Tshultrim quitte le temple par la cour, en longeant le tambour silencieux suspendu dans un coin. Le chemin le mène le long des champs désertés jusqu'à l'autre bout du village, où le bloc gris de ses chèvres l'attend au pied du noyer. 